\title{
Remarks on vacuum fluctuations around a spinning cosmic string
}

\author{
V. A. De Lorenci * and E. S. Moreira Jr. ${ }^{\dagger}$ \\ Instituto de Ciências - Escola Federal de Engenharia de Itajubá \\ Av. BPS 1303 Pinheirinho, 37500-903 Itajubá, Minas Gerais - Brazil
}

(September 21, 2000)

\begin{abstract}
We apply the point-splitting method to investigate vacuum fluctuations in the nonglobally hyperbolic background of a spinning cosmic string. Implementing renormalization by removing the Minkowski contribution it is shown that although the Green function in the literature satisfies the usual Green function equation, it leads to pathological physical results.
\end{abstract}

04.70.Dy, 04.62.+v

*Email address: lorenci@cpd.efei.br

$\dagger$ Email address: moreira@cpd.efei.br 
It has been conjectured in Ref. [1] by using general dimensional arguments that the zero-point energy could lead to a purely quantum dragging effect in the background of a spinning cosmic string. As a first step to evaluate such a possible effect, the "Feynman" Green function $G_{\mathcal{F}}\left(x, x^{\prime}\right)$ was written in Ref. [1] by assuming that the standard Schwinger proper time prescription was valid in the context of this nonglobally hyperbolic spacetime.

In this work the Green function in Ref. [1] is recast and renormalized by removing the flat spacetime contribution. Then we use the point-splitting method to calculate the "vacuum fluctuations $\left\langle\phi^{2}(x)\right\rangle, "$

$$
\left\langle\phi^{2}(x)\right\rangle=i \lim _{x^{\prime} \rightarrow x} G_{\mathcal{F}}\left(x, x^{\prime}\right),
$$

for a massless scalar field around a spinning cosmic string, showing that it diverges at an infinite countable set of spatial points, despite the fact that $G_{\mathcal{F}}\left(x, x^{\prime}\right)$ satisfies the usual Green function equation.

Throughout the text $c=\hbar=1$ and $G=1 / 4$. Our notation differs slightly from that in Ref. [1].

The spacetime around a spinning cosmic string [2] is characterized by the Minkowski line element written in cylindrical coordinates

$$
d s^{2}=d \bar{t}^{2}-d r^{2}-r^{2} d \varphi^{2}-d z^{2},
$$

and by the identification

$$
(\bar{t}, r, \varphi, z) \sim(\bar{t}+2 \pi S, r, \varphi+2 \pi \alpha, z),
$$

where the spin density and the cone parameter satisfy $S \geq 0$ and $\alpha>0$, respectively. By redefining the time coordinate as $t:=\bar{t}-S \varphi / \alpha$, Eq. (2) becomes $d s^{2}=(d t+S d \varphi / \alpha)^{2}-$ $d r^{2}-r^{2} d \varphi^{2}-d z^{2}$ and Eq. (3i) yields $(t, r, \varphi, z) \sim(t, r, \varphi+2 \pi \alpha, z)$. As the region for which $r<S / \alpha$ is inhabited by closed time-like curves the background is not globally hyperbolic and, therefore, it is not clear whether the usual tools of quantum field theory make sense in this arena [3, (1].

The Schwinger representation of the "Feynman" Green function in Ref. [1] involves an integration of the product of two Bessel functions of the first kind, which can be evaluated [5] leading to the following expression for $G_{\mathcal{F}}(x, x)$ :

$$
G_{\mathcal{F}}(r)=\int_{0}^{\infty} d t \frac{(t / i \pi)^{1 / 2}}{\alpha(4 \pi i t)^{2}} e^{-r^{2} / 2 i t} \int_{-\infty}^{\infty} d \omega e^{i t \omega^{2}} \sum_{n=-\infty}^{\infty} I_{|n+\omega S| / \alpha}\left(r^{2} / 2 i t\right),
$$

where $I_{\nu}$ denotes the modified Bessel function of the first kind. Using a convenient integral representation for $I_{\nu}$ (see expression 8.431-5 in Ref. [5]), it follows that

$$
\sum_{n=-\infty}^{\infty} I_{|n+\omega S| / \alpha}(z)=\alpha e^{z}-\frac{1}{\pi} \int_{0}^{\infty} d x e^{-z \cosh x} \sum_{n=-\infty}^{\infty} \sin (|n+\omega S| \pi / \alpha) e^{-|n+\omega S| x / \alpha},
$$

which holds for $\alpha>1 / 2$, being crucial to implementing renormalization and to performing the integration over $\omega$ in Eq. (田). [We should mention that expression (5), which can be extended to $\alpha \leq 1 / 2$, has previously been used in related contexts [6, [].] Thus considering Eq. (5), Eq. (41) becomes 


$$
\begin{aligned}
G_{\mathcal{F}}(r)= & \int_{0}^{\infty} \frac{d t}{(4 \pi i t)^{2}} \\
& -\frac{i^{3 / 2}}{16 \alpha \pi^{7 / 2}} \int_{0}^{\infty} \frac{d t}{t^{3 / 2}} e^{-r^{2} / 2 i t} \sum_{n=-\infty}^{+\infty} \int_{-\infty}^{\infty} d \omega e^{i t \omega^{2}} \sin (|n+\omega S| \pi / \alpha) \\
& \times \int_{0}^{\infty} d x e^{-\left(r^{2} / 2 i t\right) \cosh x} e^{-|n+\omega S| x / \alpha} .
\end{aligned}
$$

Now the ultraviolet divergent term (the Minkowski contribution) in Eq. (6) is dropped. By inserting $\delta[\lambda-(n+\omega S) / \alpha]$ in Eq. (6) and using Poisson's formula, we are able to perform the integration over $\omega[5]$ resulting

$$
\begin{aligned}
& G_{\mathcal{F}}(r)=\frac{1}{16 \pi^{3}} \int_{0}^{\infty} \frac{d t}{t^{2}} e^{-r^{2} / 2 i t} \\
& \times \sum_{n=-\infty}^{\infty} \int_{-\infty}^{\infty} d \lambda e^{(2 \pi n S)^{2} / 4 i t} e^{2 \pi i \alpha n \lambda} \sin (|\lambda| \pi) \int_{0}^{\infty} d x e^{-\left(r^{2} / 2 i t\right) \cosh x} e^{-|\lambda| x} .
\end{aligned}
$$

The next step is to evaluate the integrations over $\lambda$ and $t$ [5]. Considering Eq. (1) it follows that

$$
\begin{aligned}
& \left\langle\phi^{2}(r)\right\rangle=-\frac{1}{8 \pi^{2} r^{2}} \int_{0}^{\infty} d x \frac{1}{\left(\pi^{2}+x^{2}\right) \cosh ^{2}(x / 2)} \\
& -\frac{1}{4 \pi^{2} r^{2}} \int_{0}^{\infty} d x \sum_{n=1}^{\infty} \frac{x^{2}-\pi^{2}\left(4 \alpha^{2} n^{2}-1\right)}{\left[\pi^{2}(2 \alpha n+1)^{2}+x^{2}\right]\left[\pi^{2}(2 \alpha n-1)^{2}+x^{2}\right]\left[\cosh ^{2}(x / 2)-(n \pi S / r)^{2}\right]} .
\end{aligned}
$$

Finally, by considering the power series expansion of $\psi(z)$ (the logarithmic derivative of the gamma function) and its properties, the summation in Eq. (8) can be evaluated. By setting $S=0$, Eq. (8) reproduces the result for a spinless cosmic string in the literature [8], $\left\langle\phi^{2}(r)\right\rangle=\left(\alpha^{-2}-1\right) / 48 \pi^{2} r^{2}$. When $S \neq 0$, it results

$$
\begin{aligned}
& \left\langle\phi^{2}(r)\right\rangle=\frac{-1}{4 \pi^{2} \alpha r^{2}} \int_{0}^{\infty} \frac{d x}{B(x, y)} \times \\
& \left\{\left(\frac{1}{\pi}\left[4 \cosh ^{2}(x / 2)-y^{2}\left(\pi^{2}-x^{2}\right)\right] \sin (\pi / \alpha)+2 y^{2} x \sin (x / \alpha)\right) \frac{1}{\cosh (x / \alpha)-\cos (\pi / \alpha)}\right. \\
& \left.\quad+y\left[\frac{y^{2}\left(\pi^{2}+x^{2}\right)}{2 \cosh (x / 2)}-2 \cosh (x / 2)\right] \cot \left(\frac{1}{\alpha y} \cosh (x / 2)\right)\right\}
\end{aligned}
$$

where $y:=S / \alpha r$ and $B(x, y):=4 \pi^{2} y^{4} x^{2}+\left[4 \cosh ^{2}(x / 2)-y^{2}\left(\pi^{2}-x^{2}\right)\right]^{2}$. For an integer $n$, when $y=1 / n \pi \alpha$, i.e. $r=n \pi S$, an inspection reveals that Eq. (9) diverges.

These divergences may be indicating that it is not safe to use standard quantum field theory prescriptions in the context of nonglobally hyperbolic spacetimes. Therefore the Green function $G_{\mathcal{F}}\left(x, x^{\prime}\right)$ in Ref. [1] should not be used, as in Eq. (1), to investigate vacuum fluctuations. In the light of these considerations, in order to verify the quantum dragging effect conjecture in Ref. [1], one should take the cosmic string with a radius (thick cosmic string) bigger than the critical radius $S / \alpha$, such that the corresponding spacetime be globally hyperbolic and, therefore, where standard quantum field theory prescriptions are reliable. 
Before closing, a remark is in order. This work shows that pathologies arise when standard tools of second quantization are used in the background of a spinning cosmic string. It is worth recalling that standard quantum mechanics in such a background is also plagued by pathologies [9].

\section{ACKNOWLEDGMENTS}

This work was partially supported by Conselho Nacional de Desenvolvimento Cientifico e Tecnológico $(\mathrm{CNPq})$ of Brazil. 


\section{REFERENCES}

[1] G.E.A. Matsas, Phys. Rev. D 42, 2927 (1990).

[2] A. Vilenkin and E.P.S. Shellard, Cosmic Strings and Other Topological Defects (Cambridge University Press, Cambridge, England, 1994).

[3] S.A. Fulling, Aspects of Quantum Field Theory in Curved Space-time (Cambridge University Press, Cambridge, England, 1989).

[4] V.P. Frolov and I.D. Novikov, Black Hole Physics - Basic Concepts and New Developments (Kluwer Academic Publishing, Dordrecht, 1998).

[5] I.S. Gradshteyn and I.W. Ryzhik, Table of Integrals, Series, and Products (Academic Press, New York, 1980).

[6] K. Shiraishi and S. Hirenzaki, Class. Quantum Grav. 9, 2277 (1992).

[7] I. Pontual and F. Moraes, Philos. Mag. A 78, 1073 (1998).

[8] A.G. Smith, in The Formation and Evolution of Cosmic Strings, Proceedings of the Cambridge Workshop, Cambridge, England, 1989, edited by G. W. Gibbons, S. W. Hawking and T. Vachaspati (Cambridge University Press, Cambridge, England, 1990).

[9] P.S. Gerbert and R. Jackiw, Commun. Math. Phys. 124, 229 (1989). 